\title{
Polymyositis/dermatomyositis is a potential risk factor for acute respiratory failure: a pulmonary heart disease
}

\author{
Shih-Huei Syue ${ }^{1}$, Yi-Hua Chang ${ }^{1}$, Pei-Ju Shih ${ }^{1}$, Cheng-Li Lin ${ }^{2,3}$, Jun-Jun Yeh ${ }^{1,3,4}$, Chia-Hung Kao ${ }^{5,6,7,8}$ \\ ${ }^{1}$ Department of Family Medicine, Ditmanson Medical Foundation Chia-Yi Christian Hospital, Chiayi; ${ }^{2}$ Management Office for Health Data, China \\ Medical University Hospital, Taichung; ${ }^{3}$ Department of Childhood Education and Nursery, Chia Nan University of Pharmacy and Science, Tainan; \\ ${ }^{4}$ School of Chinese Medicine, ${ }^{5}$ Graduate Institute of Biomedical Sciences and School of Medicine, College of Medicine, China Medical University, \\ Taichung; 'Department of Nuclear Medicine and PET Center, and Center of Augmented Intelligence in Healthcare, China Medical University \\ Hospital, Taichung; ${ }^{7}$ Department of Bioinformatics and Medical Engineering, Asia University, Taichung; ${ }^{8}$ Center of Augmented Intelligence in \\ Healthcare, China Medical University Hospital, Taichung \\ Contributions: (I) Conception and design: JJ Yeh, CH Kao; (II) Administrative support: CH Kao; (III) Provision of study materials or patients: None; \\ (IV) Collection and assembly of data: All authors; (V) Data analysis and interpretation: All authors; (VI) Manuscript writing: All authors; (VII) Final \\ approval of manuscript: All authors. \\ Correspondence to: Chia-Hung Kao, MD, Professor. Graduate Institute of Biomedical Sciences and School of Medicine, College of Medicine, \\ China Medical University, No. 2, Yuh-Der Road, Taichung 40447. Email: d10040@mail.cmuh.org.tw; dr.kaochiahung@gmail.com; Jun-Jun Yeh, \\ MD, Professor. Department of Family Medicine, Ditmanson Medical Foundation Chia-Yi Christian Hospital, Chiayi; Department of Childhood \\ Education and Nursery, Chia Nan University of Pharmacy and Science, Tainan; School of Chinese Medicine, China Medical University, Taichung; \\ Graduate Institute of Biomedical Sciences and School of Medicine, College of Medicine, China Medical University, Taichung. \\ Email: anvin.funlan@msa.hinet.net.
}

Background: Studies on the association between polymyositis/dermatomyositis (PM/DM) and acute respiratory failure (ARF) are considerably limited. We investigated whether ARF is associated with PM/DM using a nationwide cohort study.

Methods: We identified 1,374 patients with newly diagnosed PM/DM and 13,740 comparison individuals without PM/DM (non-PM/DM) randomly selected from the general population; frequency matched by age, sex, and index year using the National Health Insurance Research Database; and followed up until the end of 2011 to measure the incidence of ARF. Cox proportional hazards regression analysis was used to measure the hazard ratio (HR) of ARF for the PM/DM cohort in comparison with the non-PM/DM cohort.

Results: The adjusted HR of ARF was 5.05 for the PM/DM cohort compared with the non-PM/DM cohort after adjusting for sex, age, comorbidities, Charlson comorbidity index (CCI) score and medicine. The risk of ARF significantly increased irrespective of age, sex, comorbidities and medicine. Meanwhile, the PM/DM cohort with comorbidities, such as cardiac disease (hypertension), pulmonary disease (chronic obstructive pulmonary disease and pneumonia), and pulmonary vascular diseases had additive effects on the incident ARF.

Conclusions: This study determined the cross-reaction of pulmonary heart disease in the PM/DM cohort with incident ARF even without comorbidities.

Keywords: Pulmonary heart disease; heart failure; polymyositis/dermatomyositis (PM/DM); acute respiratory failure (ARF); cohort study

Submitted Aug 20, 2019. Accepted for publication Nov 26, 2019.

doi: $10.21037 /$ atm.2020.01.56

View this article at: http://dx.doi.org/10.21037/atm.2020.01.56 


\section{Introduction}

Hypoxemic respiratory failure (RF) is defined as respiratory distress resulting in a $\mathrm{PaO}_{2}$ of less than $60 \mathrm{mmHg}$, despite the addition of supplemental oxygen of at least $60 \%$. Hypoxemia may be combined with high (1), normal, or low arterial carbon dioxide tension $\left(\mathrm{PaCO}_{2}\right)$ (2). Typically, this involves the pulmonary alveoli component of the pulmonary system. Hypoxemic RF is also called "lung failure" or "oxygenation failure". Its causes include hypoventilation [e.g., respiratory paralysis, asthma, and chronic obstructive pulmonary disease (COPD)] (1), diffusion impairment (e.g., severe pneumonia, interstitial fibrosis, and interstitial pulmonary edema), ventilation-perfusion (V/Q) mismatch (e.g., emphysema, alveolar pulmonary edema, and atelectasis), and intra- and extrapulmonary shunting (technically the most severe form of V/Q mismatch; e.g., lung consolidation, pulmonary embolism, and pulmonary hemorrhage) $(2,3)$.

Collagen vascular diseases such as polymyositis/ dermatomyositis (PM/DM) represent a heterogeneous group of immunologically mediated inflammatory disorders with a large variety of affected organs. Patients with PM/ DM are susceptible to respiratory involvement (4). Several different components of the respiratory system may be involved, including the airways $(5,6)$, vessels $(6,7)$, heart $(5,8)$, and parenchyma/interstitium $(6,9)$. Thoracic manifestations with the greatest clinical importance in patients with PM/ $\mathrm{DM}$ are interstitial lung disease (ILD) (10), pulmonary arterial hypertension $(11,12)$, and heart disease (10), which are responsible for the high rate of mortality and morbidity in this patient group.

Occasionally, acute respiratory failure (ARF) develops in patients with $\mathrm{PM} / \mathrm{DM}$, but the etiologies of ARF in these patients are not completely elucidated $(10,13,14)$. Comorbidities such as asthma (15), COPD (16), infection/ aspiration pneumonia $(14,17,18)$, coronary artery disease (CAD) (19), hypertension (20,21), heart failure (22), diabetes $(20,21)$, and cancer (13) are related to PM/DM. Therefore, we address an intervention of ARF with PM/ $\mathrm{DM}$ and comorbidities in this cohort study. This is the first study that focused on PM/DM presenting as ARF in the general population in the English literature.

\section{Methods}

\section{Patient and public involvement}

Taiwan's National Health Insurance (NHI) is a universal insurance program established in 1995 that covers almost $99 \%$ of the Taiwanese population. In this study, patient data were obtained from the Taiwan's National Health Insurance Research Database (NHIRD), which contains claims data from NHI, and the patient data are updated in the database every year. In the database, the disease diagnosed is recorded according to the International Classification of Diseases, Ninth Revision, Clinical Modification (ICD-9-CM).

\section{Data availability statement}

The Ministry of Health and Welfare must approve our application to access this data. Any researcher interested in accessing this dataset can submit an application form to the Ministry of Health and Welfare requesting access. The dataset used in this study is held by the Taiwan Ministry of Health and Welfare (MOHW). Please contact the staff of MOHW (Email: stcarolwu@mohw.gov.tw) for further assistance. Taiwan MOHW address: No. 488, Sec. 6, Zhongxiao E. Rd., Nangang Dist., Taipei City 115. Phone: $+886-2-8590-6848$. All relevant data are within the paper.

\section{Ethics statement}

The Research Ethics Committee of China Medical University and Hospital in Taiwan approved the study (CMUH104-REC2-115-R4). The NHIRD encrypts patient personal information to protect privacy and provides researchers with anonymous identification numbers associated with relevant claims information, including sex, date of birth, medical services received, and prescriptions. Therefore, patient consent is not required to access the NHIRD.

\section{Study population}

This study used a retrospective population-based cohort design. A cohort of newly diagnosed PM/DM (ICD-9-CM: 710.3 and 710.4) from 2000 to 2009 was established. The baseline of the PM/DM cohort was the initial PM/DM diagnosis date. Individuals without PM/DM were randomly selected, frequency matched by sex and age (per 5 years) in a 1:10 ratio, and used as the non-PM/DM comparison cohort. The baseline of the non-PM/DM comparison cohort was randomly assigned a month and day and the same year of the matched PM/DM cases.

The ARF defined as the hypoxaemic (arterial oxygen 
tension, $\mathrm{PaO}_{2}<60 \mathrm{mmHg}$ ) with or without hypercapnia (arterial carbon dioxide tension, $\mathrm{PaCO}_{2}>50 \mathrm{mmHg}$ ). The $\mathrm{ARF}$ develops within minutes or hours, thus the $\mathrm{pH}<7.3$ and the value of the bicarbonate ion is normal. Meanwhile, these patients with loss of the ability to ventilate adequately or to provide sufficient oxygen to the blood and systemic organs (23-25). Owing to the incident ARF was enrolled into the catastrophic diseases, the policy of definition the ARF in Taiwan NHIRD is strict $(26,27)$. The principal outcome was ARF occurrence (ARF, ICD-9-CM: 518.81). Patients who had experienced incident ARF before the baseline were excluded. Follow-up was terminated upon the observation of an ARF event or at the end of the year (December 31, 2011).

ARF comorbidities recorded included the disease history before the baseline and were considered as potential confounding factors. We categorized Charlson-comorbidity index (CCI) into 4 levels: $0,1,2$, and 3 or more. These comorbidities included asthma (ICD-9-CM: 493, 494, and A324), hypertension (ICD-9-CM: 401-405), DM (ICD9-CM: 250), COPD (ICD-9-CM: 491, 492, and 496), pneumonia (ICD-9-CM: 480-487), cancer (ICD-9-CM: 140-208), CAD (ICD-9-CM: 410-414), heart failure (ICD9-CM: 428), and pulmonary vascular disease (PVD, ICD9-CM: 415-417). In addition, oral steroid was analyzed between the PM/DM patients and the non-PM/DM cohort. Methotrexate was analyzed among PM/DM cohort.

\section{Statistical analysis}

We demonstrated the mean and standard deviation (SD) for age at baseline and presented the frequency and percentage for sex and baseline comorbidities. Chi-squared test was used to analyze categorical variables, and Student's $t$-test was used to assess continuous variables comparing the PM/ $\mathrm{DM}$ and non-PM/DM cohorts in terms of the baseline demographic status. The incidence density rate of incident ARF for both cohorts were calculated as the number of incident ARF occurrences divided by the total sum of the follow-up year (per 10,000 person-years), the Kaplan-Meier method was used to estimate the cumulative incidence ARF of the studied subjects during the follow-up period, and the log-rank test was used to test the differences between both curves. Cox proportional hazards regression was used to estimate the hazard ratios (HRs) and 95\% confidence intervals (CIs) of incident ARF risk in the PM/DM and non-PM/DM cohorts.

All statistical analyses were performed using the SAS 9.4 statistical package (SAS Institute Inc., NC, USA). A P value of $<0.05$ in two-tailed tests was considered significant.

\section{Results}

\section{Characteristics of the study participants}

We evaluated 1,374 PM/DM patients and 13,740 non-PM/ DM individuals (Table 1). Because age and sex were matched, no statistically significant differences in age $(49.6 \pm 14.4$ years $)$ and sex ratio (male: $31.1 \%$ ) were observed between the two cohorts. Except for hypertension and diabetes, the patients with $\mathrm{PM} / \mathrm{DM}$ had a higher percentage of patients with comorbidities than the non-PM/DM cohort (all $\mathrm{P}$ values $<0.05)$. Most of the PM/DM patients had the number of CCI score of $\geq 1(52.8 \%)$. The medication used in the both $\mathrm{PM} / \mathrm{DM}$ and non-PM/DM cohorts was oral steroid (98.8\% vs. $69.5 \%$ ). In the PM/DM cohort, approximately $65 \%$ of the patients were used of methotrexate.

\section{The incidence and cumulative incidence of ARF between the PM/DM and non-PM/DM cohorts}

The incidences of ARF were 229.44 and 37.08 per 10,000 person-years in the PM/DM and non-PM/DM cohorts, respectively (Table 2). We also observed that the curve of the ARF cumulative incidence for the PM/DM cohort was greater than that for the non-PM/DM cohort (Figure 1; P for the log-rank test $<0.0001$ ).

\section{Adjusted HR of ARF}

After adjusting for age, sex, CCI score, comorbidities, and medicine of oral steroid, the results showed that the PM/ DM patients had higher risk of ARF (HR: 5.05 ; $95 \%$ CI: 4.08-6.25) than the non-PM/DM individuals. Compared with patients aged 45 years and younger, the risk of ARF development is 2.81-fold higher in those aged 45-64 years (95\% CI: 2.01-3.92), and 9.11-fold higher in those aged 65 years and older (95\% CI: 6.39-13.00). Multivariable models showed that ARF was associated with men, CCI score, hypertension, diabetes, COPD, pneumonia, and PVD.

\section{ARF in relation to the average frequency of hospital and clinical visits}

As the non-PM/DM cohort as the reference, the number of hospitalizations and clinical visits of $\geq 16$ (HR: 12.2; 95\% 
Table 1 Baseline demographic status and comorbidity compared between the non-PM/DM and PM/DM cohorts

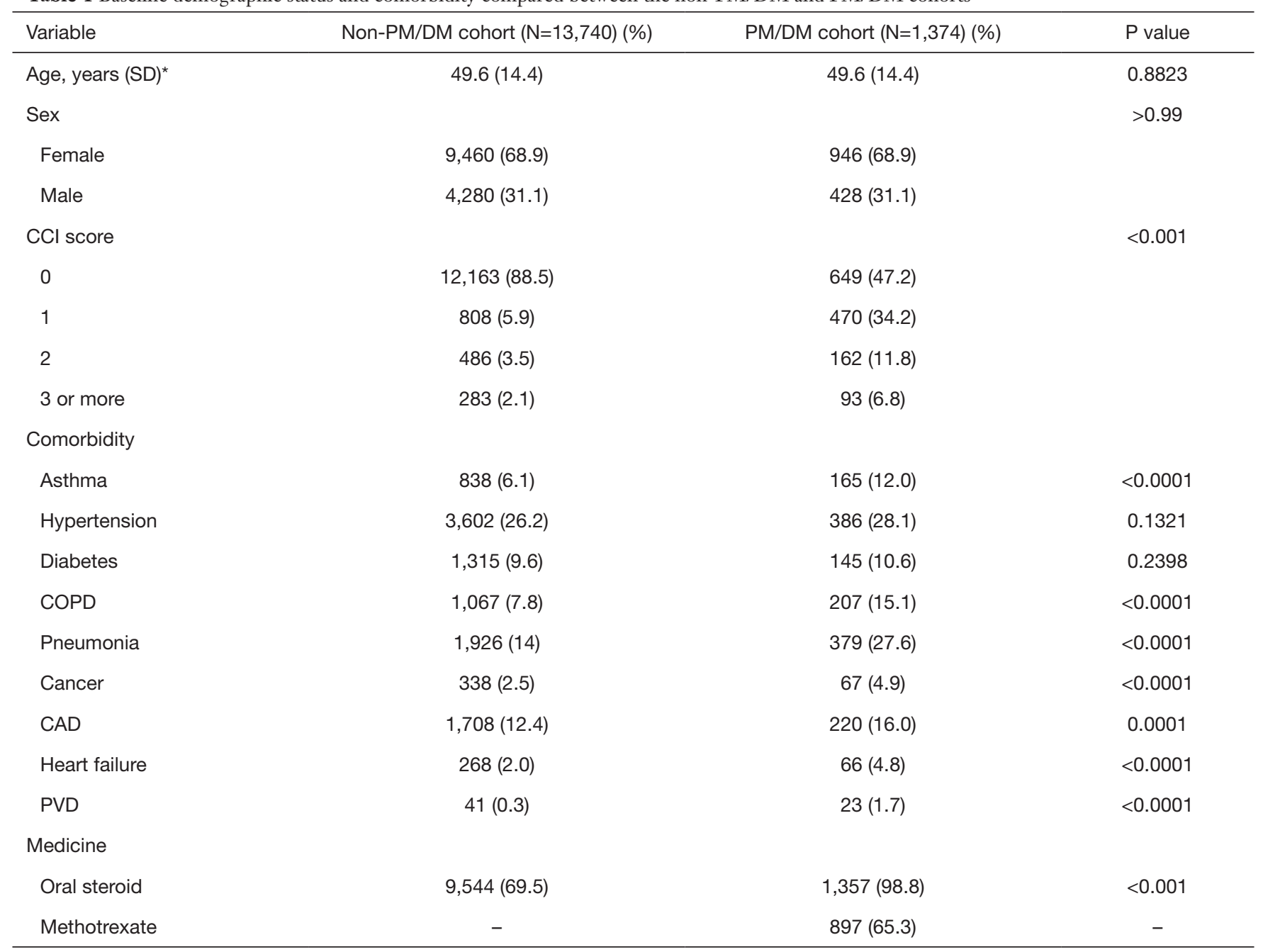

*, $t$-test. PM/DM, polymyositis/dermatomyositis; SD, standard deviation; CCl score, Charlson comorbidity index score; COPD, chronic obstructive pulmonary disease; CAD, coronary artery disease; PVD, pulmonary vascular disease.

CI: 9.58-15.50) had the highest risk of ARF compared with the frequencies of hospital and clinical visits of $8-15$ times (HR: 2.59; 95\% CI: $1.81-3.70$ ) and 8 times (HR: 1.78 ; $95 \%$ CI: 1.10-2.90) (Table 3). The results also make known that increased average frequency of hospital and clinic visits was significantly increased risk of ARF ( $\mathrm{P}$ for trend $<0.0001$ ).

\section{Interaction between the PM/DM cohort and comorbidity}

Regarding the interaction effects of PM/DM and comorbidity on ARF risk (Table 4), the PM/DM patients with hypertension (HR: 4.01; 95\% CI: 2.99-5.36), COPD (HR: 3.04; 95\% CI: 2.04-4.54), and PVD (HR: 18.4; 95\% CI: 2.21-153.8) had higher risk of ARF than the non-PM/
$\mathrm{DM}$ patients with these comorbidities ( $\mathrm{P}$ for interaction $<0.05)$, except asthma, diabetes, pneumonia, cancer, CAD, and heart failure. These results display the joint effects of $\mathrm{PM} / \mathrm{DM}$ and comorbidity on incident ARF risk.

\section{Discussion}

The first important finding of this study suggests that the incidence of ARF in the PM/DM cohort was higher than that in the non-PM/DM cohort (HR: 5.05; 95\% CI: 4.08-6.25). ARF is a major cause of death in the PM/DM cohort $(5,6,28)$. Santo et al. found that the frequency of death due to ARF in the PM/DM cohort was as high as $32.4 \%$ (29), which supports the finding of the current study. 
Table 2 Incidence of acute respiratory failure for the study cohort and multivariate Cox proportional hazards regression analysis measuring hazard ratio for respiratory failure

\begin{tabular}{|c|c|c|c|c|c|}
\hline Variable & Event & PYs & Rate & Crude HR $(95 \% \mathrm{Cl})$ & Adjusted HR (95\% Cl) \\
\hline \multicolumn{6}{|l|}{ PM/DM } \\
\hline No & 335 & 90,336 & 37.08 & Ref & Ref \\
\hline Yes & 171 & 7,453 & 229.44 & $6.10(5.08-7.34)$ & $5.05(4.08-6.25)$ \\
\hline \multicolumn{6}{|l|}{ Age group } \\
\hline $45-64$ years & 188 & 44,773 & 41.99 & $3.64(2.63-5.03)$ & $2.81(2.01-3.92)$ \\
\hline$\geq 65$ years & 273 & 13,996 & 195.06 & 16.77 (12.23-22.99) & $9.11(6.39-13.00)$ \\
\hline \multicolumn{6}{|l|}{ Sex } \\
\hline Female & 284 & 67,731 & 41.93 & Ref & Ref \\
\hline 0 & 219 & 85,124 & 25.7 & Ref & Ref \\
\hline 1 & 138 & 7,664 & 180.1 & 6.97 (5.63-8.62) & $2.52(2.00-3.18)$ \\
\hline 2 & 76 & 3,377 & 225.0 & $8.67(6.67-11.3)$ & $3.24(2.37-4.43)$ \\
\hline 3 or more & 73 & 1,623 & 450.0 & $17.20(13.20-22.40)$ & $4.45(3.21-6.17)$ \\
\hline \multicolumn{6}{|l|}{ Comorbidity } \\
\hline \multicolumn{6}{|l|}{ Asthma } \\
\hline No & 422 & 91,998 & 45.87 & Ref & Ref \\
\hline Yes & 84 & 5791 & 145.06 & $3.15(2.49-3.98)$ & $1.01(0.78-1.31)$ \\
\hline Yes & 129 & 8,550 & 150.88 & $3.55(2.9-4.33)$ & $1.48(1.19-1.83)$ \\
\hline \multicolumn{6}{|l|}{ COPD } \\
\hline No & 371 & 90,671 & 40.92 & Ref & Ref \\
\hline Yes & 135 & 7,118 & 189.66 & $4.60(3.78-5.61)$ & $1.25(1.00-1.58)$ \\
\hline \multicolumn{6}{|l|}{ Pneumonia } \\
\hline No & 356 & 84,480 & 42.14 & Ref & Ref \\
\hline Yes & 150 & 13,309 & 112.71 & $2.67(2.21-3.24)$ & $1.26(1.03-1.55)$ \\
\hline \multicolumn{6}{|l|}{ Cancer } \\
\hline No & 473 & 95,836 & 49.35 & Ref & Ref \\
\hline Yes & 33 & 1,952 & 169.03 & $3.33(2.34-4.74)$ & $0.88(0.59-1.31)$ \\
\hline
\end{tabular}

Table 2 (continued) 
Table 2 (continued)

\begin{tabular}{|c|c|c|c|c|c|}
\hline Variable & Event & PYs & Rate & Crude HR $(95 \% \mathrm{Cl})$ & Adjusted HR (95\% Cl) \\
\hline No & 334 & 86,099 & 38.79 & Ref & Ref \\
\hline Yes & 172 & 11,690 & 147.13 & $3.78(3.14-4.54)$ & $0.90(0.72-1.11)$ \\
\hline \multicolumn{6}{|c|}{ Heart failure } \\
\hline Yes & 59 & 1,733 & 340.45 & $7.20(5.48-9.44)$ & $0.75(0.52-1.07)$ \\
\hline \multicolumn{6}{|l|}{ PVD } \\
\hline No & 491 & 97,476 & 50.37 & Ref & Ref \\
\hline Yes & 15 & 313 & 479.23 & $9.36(5.60-15.65)$ & $1.96(1.15-3.34)$ \\
\hline No & 85 & 30,419 & 27.94 & Ref & Ref \\
\hline Yes & 421 & 67,370 & 62.50 & $2.23(1.77-2.82)^{\star \star \star}$ & $1.04(0.81-1.34)$ \\
\hline
\end{tabular}

Adjusted model was mutually adjusted. ${ }^{\star \star *}, \mathrm{P}<0.001$. Rate, per 10,000 person-years; PYs, person-years; Cl, confidence interval; HR, hazard ratio; PM/DM, polymyositis/dermatomyositis; CCl score, Charlson comorbidity index score; COPD, chronic obstructive pulmonary disease; CAD, coronary artery disease; PVD, pulmonary vascular disease.

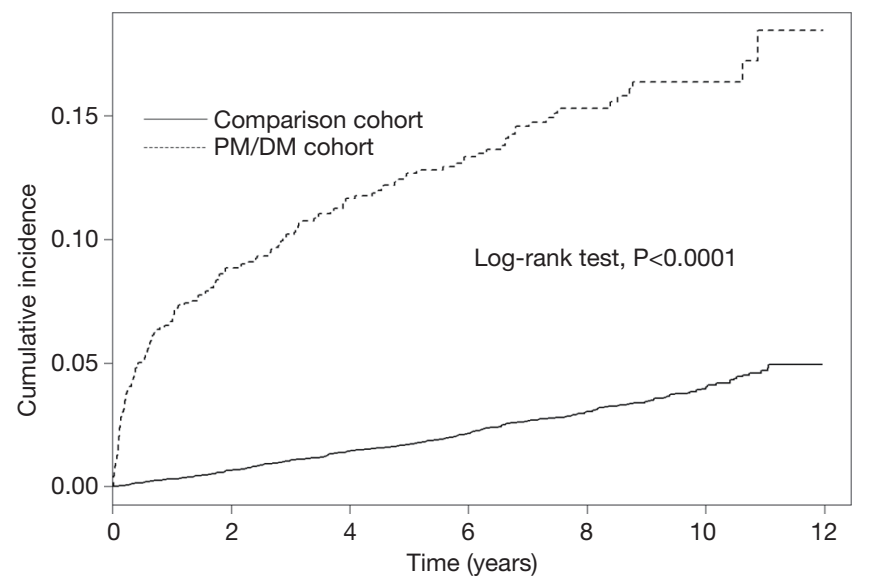

Figure 1 The cumulative incidence of developing acute respiratory failure. Comparison cohort vs. PM/DM cohort.

The age-stratified effects of the PM/DM cohort on ARF development were high in patients aged $45-64$ years (HR: 2.88 ) and $>65$ years (HR: 9.83). The higher CCI score such as $>3$ was associated with a high risk of ARF in the PM/DM cohort (adjusted HR is high up to 4.45), which is similar to Santo et al.'s finding that the risk of death is higher when patients in the PM/DM cohort exhibit neoplasm. The mean age of $61.50 \pm 13.66$ years in the PM/DM cohort with cancer supported this finding (29). Meanwhile, Marie et al., study revealed that the PM/DM with the severe ILD having the higher age (mean age, 62 years) and mortality $(47.1 \%)$ support our result (30).

The etiology of RF includes cardiac origin diseases such as hypertension (21), heart failure, and CAD and pulmonary origin diseases such as COPD (hypoventilation) and pneumonia (diffusion impairment). Therefore, the primary pulmonary complication of $\mathrm{PM} / \mathrm{DM}$ such as pneumonia and the primary cardiac complication such as hypertensionrelated heart failure (V/Q mismatch) contribute to the incident $\mathrm{ARF}$ and death. These findings imply that PM/DM itself $(5,31,32)$ is a predisposing factor for ARF, even in the absence of comorbidities (11,33). Similarly, Santo et al. (29) reported that respiratory and circulatory disorders (28) were the principal associated causes of ARF in PM/DM patients $(10,14)$. As shown in Table 4, the PM/DM cohort without any comorbidities was associated with higher risk of ARF than the non-PM/DM cohort, which is in line with these previous findings. Furthermore, the asthma, diabetes, pneumonia, heart failure, CAD and cancer without additive effects on the incident ARF support these results also (Table 4).

The late course of the PM/DM with severity of the ILD receiving the higher dose of the steroid (34), the anti- 
Table 3 Association between the incidence of acute respiratory failure for different levels of average frequency of hospital and clinical visits for $\mathrm{PM} / \mathrm{DM}$ and estimation of the risk of respiratory failure using multivariate Cox proportional hazards regression analysis

\begin{tabular}{|c|c|c|c|c|c|}
\hline $\begin{array}{l}\text { Average frequency of PM/DM, per } \\
\text { year }\end{array}$ & Event & PYs & Rate & Crude HR $(95 \%$ Cl) & Adjusted HR (95\% Cl) \\
\hline Non-PM/DM cohort & 335 & 90,336 & 37.08 & Ref & Ref \\
\hline$<8$ & 18 & 2,609 & 68.99 & $1.86(1.16-2.99)$ & $1.78(1.10-2.90)$ \\
\hline
\end{tabular}

Model adjusted for age, sex, CCI score, asthma, hypertension, diabetes, COPD, pneumonia, cancer, CAD, heart failure, and PVD, and medicine of oral steroid. PM/DM, polymyositis/dermatomyositis; rate, per 10000 person-years; PYs, person-years; Cl, confidence interval; $\mathrm{HR}$, hazard ratio; CCI score, Charlson comorbidity index score; COPD, chronic obstructive pulmonary disease; CAD, coronary artery disease; PVD, pulmonary vascular disease.

inflammatory may attenuate the airway inflammation; thus, these groups patients with asthma didn't have the association and addictive effects on the incident ARF (Table 2) $(32,35)$. Similar to the asthma patients, the cancer was associated with the late stage of the long-course of the PM/ $\mathrm{DM}$ (36). The steroid may have beneficence on the PM/DM with the cancer with ILD-ARF $(37,38)$. Thus, these groups patients receiving the higher dose steroid without association and additive effects on the incident ARF (Tables 2,4). These hypotheses warrant further researches.

PM/DM with comorbidities such as COPD, hypertension and PVD were associated with an increased risk of incident ARF. COPD (1) contributes to ARF owing to the obstructive airway. Hypertension may be a precipitated factor of heart failure with interstitial edema (diffusion impairment) (2) and contribute to incident ARF. Diabetes (21) is also a disease with airway inflammation such as COPD (39) and atherosclerosis of vessel such as hypertension (40). This implies that diabetes is associated with cardiopulmonary complications in the deterioration of the PM/DM cohort $(41)$ such as acute $(7,8)$ or chronic pulmonary heart disease (42). These findings suggest that diabetes was associated with the incident ARF in the PM/ DM cohort. However, the PM/DM patients primarily have the insulin resistance (43). Therefore, the diabetes having not the additive effects on the incident ARF (Table 4).

Primary cardiac complications in the PM/DM cohort included congestive heart failure (V/Q mismatch) resulting from primary cardiomyopathy, dysrhythmias, atrioventricular conduction disturbances, sick sinus syndrome (44), and an example of PVD with pulmonary heart reaction such as cor pulmonale (11). In addition, respiratory complications in the PM/DM cohort included recurrent aspiration pneumonia resulting from pharyngeal muscle involvement by the myositic process, and the involvement of respiration muscles can lead to ARF. Therefore, the heart and lungs (45) displayed a primary cross-reaction in the PM/DM cohort with ARF even without comorbidities $(8,41)$. We found that pneumonia was associated with the ARF and it is without additive effects on the development of the incident ARF in line with these results. Meanwhile, the heart failure and CAD without additive effects on the incident ARF support these speculations also.

The frequency of admission of the PM/DM cohort increased the risk of ARF. The higher frequency of pulmonary artery inflammation in the PM/DM cohort, higher CCI increased the risk of primary pulmonary artery occlusion such as chronic pulmonary embolism combined with heart failure (an example of pulmonary heart reaction) (7). Meanwhile, the recurrence of inflammation increased the primary chronic inflammation of the interstitium of the lung and parenchymal lesion such as pneumonia (4). The combined effects of primary chronic pulmonary embolism (V/Q mismatch) and chronic inflammation of the interstitium of the lung (diffusion impairment) contribute to PVD (e.g., primary pulmonary artery hypertension) after primary heart failure and pneumonia (4). Similar to these previous findings, systematic review data showed that heart failure is the main cause of pulmonary artery hypertension (46), primary and chronic inflammation of cardiomyopathy contributed to fibrosis myopathy of the heart in the PM/DM cohort (44). These bi-directional speculations explain the primary chronic inflammation in 
Table 4 Incidence of acute respiratory failure for the study cohort stratified by comorbidities and estimation of the risk of respiratory failure for different levels of comorbidities using multivariate Cox proportional hazards regression analysis

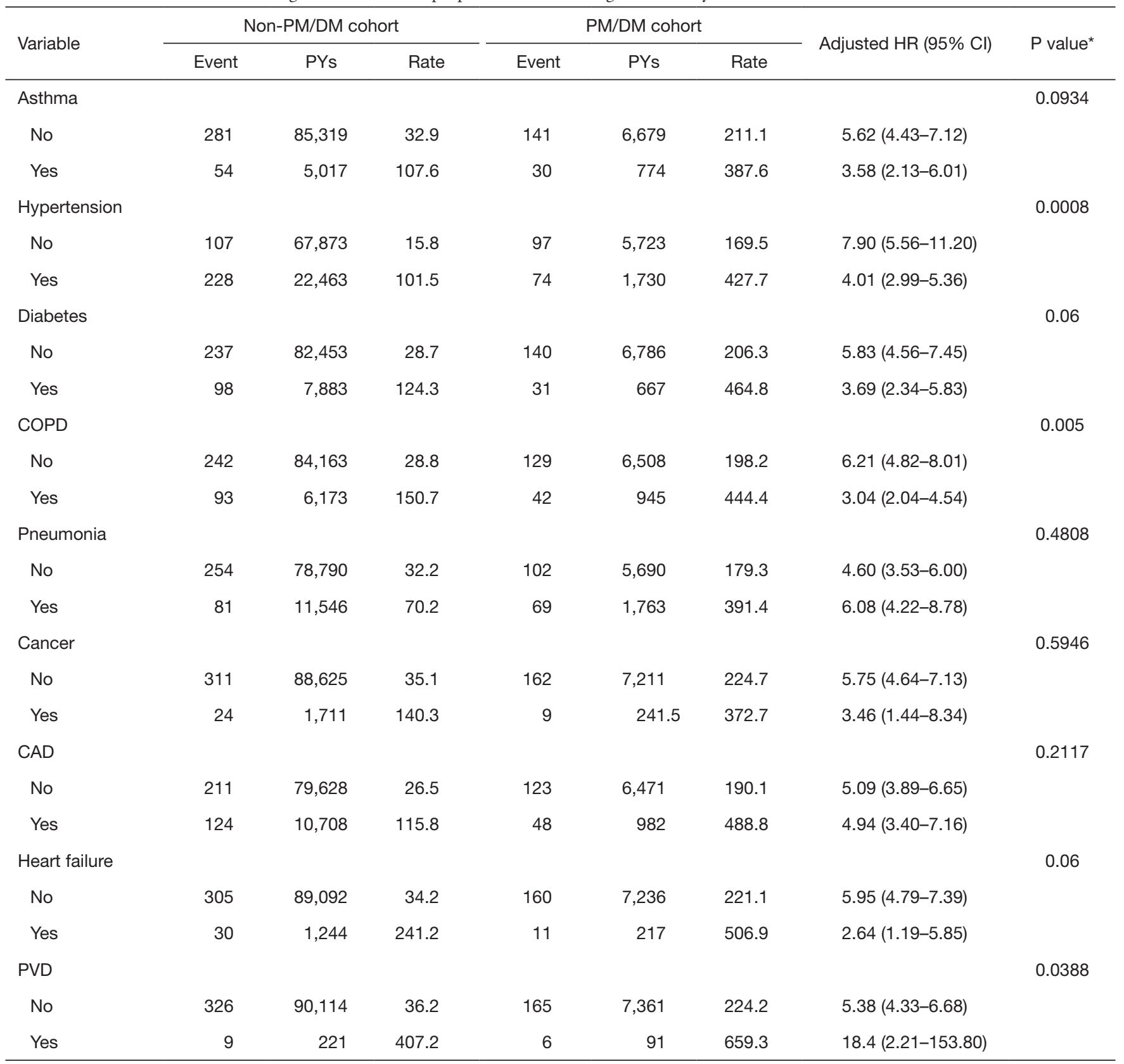

Adjusted for age, sex, CCl score, and medicine of oral steroid. ${ }^{*}$, P for interaction. PM/DM, polymyositis/dermatomyositis; rate, per 10,000 person-years; PYs, person-years; CI, confidence interval; HR, hazard ratio; COPD, chronic obstructive pulmonary disease; CAD, coronary artery disease; PVD, pulmonary vascular disease; $\mathrm{CCl}$ score, Charlson comorbidity index score.

the chronic reaction of the heart and lungs in the PM/DM cohort during follow-up, especially in patients with a high frequency of hospitalization, thus contributing to incident ARF (44). Furthermore, diabetes with insulin resistance (40) is associated with cardiopulmonary diseases (41) in the progressive course of the PM/DM cohort $(5,20,21)$. These findings highlight the primary cardiopulmonary reaction $(41,46)$ in chronic pulmonary heart diseases with a high frequency of admission among the PM/DM with poor control cohort, especially patients elderly, male and with 
comorbidities (e.g., COPD, hypertension, PVD) (8). In summary, this pulmonary-heart reaction in the PM/DM cohort is associated with the incident $\operatorname{ARF}(23,32,47)$.

In a previous study, ARF in the PM/DM cohort focuses on the myopathy of patients $(48,49)$. The long-term outcome of the PM/DM cohort is associated with lung disease and inflammatory myopathies (50). The important finding of this study is that cardiac and pulmonary origins play a critical role in incident ARF development in the PM/ DM cohort (33). Therefore, this study implies the crossreaction of the heart and lungs $(32,41,46)$ in the event of ARF among the PM/DM cohort. Furthermore, bridging the pulmonary diseases (e.g., COPD), heart disease (e.g., hypertension) $(8,32,41,42)$ and PVD in the PM/DM cohort with incident ARF close together $(23,31-33,47,51)$.

\section{Limitation}

Several limitations must be considered when interpreting these findings. The NHIRD does not provide detailed lifestyle information, such as smoking, body mass index (BMI), and physical activity, all of which were potential confounding factors in this study. In this study, we replace smoking with COPD and replace the BMI with diabetes. Although PM/DM treatment and the lifestyle modification of PM/DM patients implicate these factors in accelerated atherosclerosis vessel and airway inflammation in PM/ DM. Additionally, information on PM/DM severity such as CCI were enrolled into analysis. However, disease activity, functional impairment, and physical damage were unavailable in our data. We only use the drug data such as steroids to adjust for the outcomes of interest. The other drugs use in the PM/DM cohort could be another limitation of this study. Despite our meticulous study design for controlling confounding factors, a key limitation of this study is the potential for bias caused by possible unmeasured or unknown confounders.

\section{Strength}

The strength of this study is that it provides a nationwide population-based cohort longitudinal study on ARF risk in Asian people with PM/DM. The analysis of PM/DM is based on the inpatient claims data and the catastrophic illnesses registry. The cohort study in the general population is similar to the "real world" (52) and avoids the bias of diagnosis and follow-up. These findings can be generalized to the general population.

\section{Conclusions}

This study determined the cross-reaction of pulmonary heart disease in the PM/DM cohort with incident ARF even without comorbidities.

\section{Acknowledgments}

Funding: This study is supported in part by Taiwan Ministry of Health and Welfare Clinical Trial and Research Center of Excellence (MOHW108TDU-B-212-133004); China Medical University Hospital (DMR-107-192, CMU107-ASIA-19); Academia Sinica Stroke Biosignature Project (BM10701010021); MOST Clinical Trial Consortium for Stroke (MOST 108-2321B-039-003-); Tseng-Lien Lin Foundation, Taichung; and Katsuzo and Kiyo Aoshima Memorial Funds, Japan. The funders had no role in the study design, data collection and analysis, the decision to publish, or preparation of the manuscript.

\section{Footnote}

Conflicts of Interest: The authors have no conflicts of interest to declare.

Ethical Statement: The authors are responsible for all aspects of the work in ensuring that questions related to the accuracy or integrity of any part of the work are appropriately investigated and resolved. The Research Ethics Committee of China Medical University and Hospital in Taiwan approved the study (CMUH104REC2-115-R4). The NHIRD encrypts patient personal information to protect privacy and provides researchers with anonymous identification numbers associated with relevant claims information, including sex, date of birth, medical services received, and prescriptions. Therefore, patient consent is not required to access the NHIRD.

Open Access Statement: This is an Open Access article distributed in accordance with the Creative Commons Attribution-NonCommercial-NoDerivs 4.0 International License (CC BY-NC-ND 4.0), which permits the noncommercial replication and distribution of the article with the strict proviso that no changes or edits are made and the original work is properly cited (including links to both the formal publication through the relevant DOI and the license). See: https://creativecommons.org/licenses/by-nc-nd/4.0/. 


\section{References}

1. Cham GW, Tan WP, Earnest A, et al. Clinical predictors of acute respiratory acidosis during exacerbation of asthma and chronic obstructive pulmonary disease. Eur J Emerg Med 2002:9:225-32.

2. Greene KE, Peters JI. Pathophysiology of acute respiratory failure. Clin Chest Med 1994:15:1-12.

3. Gunning KEJ. Pathophysiology of Respiratory Failure and Indications for Respiratory Support. Surgery (Oxford) 2003:21:72-6.

4. Antoniou KM, Margaritopoulos G, Economidou F, et al. Pivotal clinical dilemmas in collagen vascular diseases associated with interstitial lung involvement. Eur Respir J 2009:33:882-96.

5. Schwarz MI. Pulmonary and cardiac manifestations of polymyositis-dermatomyositis. J Thorac Imaging 1992:7:46-54.

6. Fathi M, Lundberg IE, Tornling G. Pulmonary complications of polymyositis and dermatomyositis. Semin Respir Crit Care Med 2007:28:451-8.

7. Carruthers EC, Choi HK, Sayre EC, et al. Risk of deep venous thrombosis and pulmonary embolism in individuals with polymyositis and dermatomyositis: a general population-based study. Ann Rheum Dis 2016;75:110-6.

8. Hirano T, Nakanishi M, Kawaguchi M, et al. Dermatomyositis complicated with heart and lung disease and died of rapidly progressive respiratory failure. Nihon Naika Gakkai Zasshi 2001:90:693-5.

9. Mathiesen PR, Buchvald F, Nielsen KG, et al. Pulmonary function and autoantibodies in a long-term follow-up of juvenile dermatomyositis patients. Rheumatology (Oxford) 2014:53:644-9.

10. Murabayashi K, Saito E, Okada S, et al. Prognosis of life in polymyositis/dermatomyositis. Ryumachi 1991:31:391-7.

11. Hallowell RW, Ascherman DP, Danoff SK. Pulmonary manifestations of polymyositis/dermatomyositis. Semin Respir Crit Care Med 2014:35:239-48.

12. Prakash UB. Respiratory complications in mixed connective tissue disease. Clin Chest Med 1998:19:733-46, ix.

13. Hidano A, Torikai S, Uemura T, et al. Malignancy and interstitial pneumonitis as fatal complications in dermatomyositis. J Dermatol 1992:19:153-60.

14. Lee J, Yim JJ, Yang SC, et al. Outcome of patients with connective tissue disease requiring intensive care for respiratory failure. Rheumatol Int 2012:32:3353-8.

15. Bazarnaia NS. Development of dermatomyositis in a patient with bronchial asthma. Ter Arkh 1971:43:105-6.
16. Pouessel G, Deschildre A, Le Bourgeois M, et al. The lung is involved in juvenile dermatomyositis. Pediatr Pulmonol 2013:48:1016-25.

17. Marie I, Menard JF, Hachulla E, et al. Infectious complications in polymyositis and dermatomyositis: a series of 279 patients. Semin Arthritis Rheum 2011:41:48-60.

18. Sherer Y, Shepshelovich D, Shalev T, et al. Outcome of patients having dermatomyositis admitted to the intensive care unit. Clin Rheumatol 2007:26:1851-5.

19. Lin YN, Lin CL, Chang KC, et al. Increased subsequent risk of acute coronary syndrome for patients with dermatomyositis/polymyositis: a nationwide populationbased retrospective cohort study. Scand J Rheumatol 2015;44:42-7.

20. Diederichsen LP, Diederichsen AC, Simonsen JA, et al. Traditional cardiovascular risk factors and coronary artery calcification in adults with polymyositis and dermatomyositis: a Danish multicenter study. Arthritis Care Res (Hoboken) 2015;67:848-54.

21. Souza FH, Levy-Neto M, Shinjo SK. Prevalence of clinical and laboratory manifestations and comorbidities in polymyositis according to gender. Rev Bras Reumatol 2011:51:428-83.

22. Zuo C, Wei XD, Ye YL, et al. Risk factors associated with cardiac involvement in patients with dermatomyositis/ polymyositis. Sichuan Da Xue Xue Bao Yi Xue Ban 2013:44:801-4, 809.

23. Cortes-Puentes GA, Oeckler RA, Marini JJ. Physiologyguided management of hemodynamics in acute respiratory distress syndrome. Ann Transl Med 2018:6:353.

24. Roussos C, Koutsoukou A. Respiratory failure. Eur Respir J Suppl 2003:47:3s-14s.

25. Yeh JJ. Predictors of Initial Smear-Negative Active Pulmonary Tuberculosis with Acute Early Stage Lung Injury by High-Resolution Computed Tomography and Clinical Manifestations: An Auxiliary Model in Critical Patients. Sci Rep 2019;9:4527.

26. Yeh JJ, Syue SH, Lin CL, et al. Statin use and Vital Organ Failure in Patients With Asthma-Chronic Obstructive Pulmonary Disease Overlap: A Time-Dependent Population-Based Study. Front Pharmacol 2019;10:889.

27. Hsieh CY, Su CC, Shao SC, et al. Taiwan's National Health Insurance Research Database: past and future. Clin Epidemiol 2019:11:349-58.

28. Danieli MG, Gambini S, Pettinari L, et al. Impact of treatment on survival in polymyositis and dermatomyositis. A single-centre long-term follow-up study. Autoimmun Rev 2014:13:1048-54. 
29. Santo AH, Souza JM, Pinheiro CE, et al. Trends in dermatomyositis- and polymyositis-related mortality in the state of Sao Paulo, Brazil, 1985-2007: multiple cause-ofdeath analysis. BMC Public Health 2010:10:597.

30. Marie I, Hatron PY, Dominique S, et al. Short-term and long-term outcomes of interstitial lung disease in polymyositis and dermatomyositis: A series of 107 patients. Arthritis Rheum 2011:63:3439-47.

31. De Paepe B. Vascular changes and perifascicular muscle fiber damage in dermatomyositis: another question of the chicken or the egg that is on our mind. Ann Transl Med 2017:5:22.

32. Peng JM, Du B, Wang Q, et al. Dermatomyositis and Polymyositis in the Intensive Care Unit: A Single-Center Retrospective Cohort Study of 102 Patients. PLoS One 2016:11:e0154441.

33. Yang SH, Chang C, Lian ZX. Polymyositis and dermatomyositis - challenges in diagnosis and management. Journal of Translational Autoimmunity 2019;2:100018.

34. Wu C, Wang Q, He L, et al. Hospitalization mortality and associated risk factors in patients with polymyositis and dermatomyositis: A retrospective case-control study. PLoS One 2018:13:e0192491.

35. Jantz MA, Sahn S. Corticosteroids in Acute Respiratory Failure. Am J Respir Crit Care Med 1999:160:1079-100.

36. Kohsaka H, Mimori T, Kanda T, et al. Treatment consensus for management of polymyositis and dermatomyositis among rheumatologists, neurologists and dermatologists. Neurol Clin Neurosci 2019;7:3-21.

37. Kim HJ, Cha SI, Kim CH, et al. Risk factors of postoperative acute lung injury following lobectomy for nonsmall cell lung cancer. Medicine (Baltimore) 2019:98:e15078.

38. Kim S, Oh IJ, Park SY, et al. Corticosteroid therapy against treatment-related pulmonary toxicities in patients with lung cancer. J Thorac Dis 2014:6:1209-17.

39. Mirrakhimov AE. Chronic obstructive pulmonary disease and glucose metabolism: a bitter sweet symphony. Cardiovasc Diabetol 2012:11:132.

40. Zoungas S, Woodward M, Li Q, et al. Impact of age, age at diagnosis and duration of diabetes on the risk of macrovascular and microvascular complications and death in type 2 diabetes. Diabetologia 2014:57:2465-74.

41. Han MK, McLaughlin VV, Criner GJ, et al. Pulmonary diseases and the heart. Circulation 2007:116:2992-3005.

42. Choudhary G, Jankowich M, Wu WC. Elevated pulmonary artery systolic pressure predicts heart failure admissions in African Americans: Jackson Heart Study. Circ Heart Fail 2014:7:558-64.

43. de Oliveira DS, Guimarães MS, Shinjo SK. Insulin resistance is increased in adult patients with dermatomyositis. MedicalExpress (São Paulo, online) 2018;5:mo18003.

44. Zhang L, Wang GC, Ma L, et al. Cardiac involvement in adult polymyositis or dermatomyositis: a systematic review. Clin Cardiol 2012:35:686-91.

45. Mereles D, Ehlken N, Kreuscher S, et al. Exercise and respiratory training improve exercise capacity and quality of life in patients with severe chronic pulmonary hypertension. Circulation 2006:114:1482-9.

46. Dzudie A, Kengne AP, Thienemann F, et al. Predictors of hospitalisations for heart failure and mortality in patients with pulmonary hypertension associated with left heart disease: a systematic review. BMJ Open 2014:4:e004843.

47. Sipmann FS, Santos A, Tusman G. Heart-lung interactions in acute respiratory distress syndrome: pathophysiology, detection and management strategies. Ann Transl Med 2018;6:27.

48. Oh TH, Brumfield KA, Hoskin TL, et al. Dysphagia in inflammatory myopathy: clinical characteristics, treatment strategies, and outcome in 62 patients. Mayo Clin Proc 2007:82:441-7.

49. Selva-O'Callaghan A, Sanchez-Sitjes L, Munoz-Gall $\mathrm{X}$, et al. Respiratory failure due to muscle weakness in inflammatory myopathies: maintenance therapy with home mechanical ventilation. Rheumatology (Oxford) 2000:39:914-6.

50. Selva-O'Callaghan A, Labrador-Horrillo M, MunozGall X, et al. Polymyositis/dermatomyositis-associated lung disease: analysis of a series of 81 patients. Lupus 2005:14:534-42.

51. Repessé X, Vieillard-Baron A. Right heart function during acute respiratory distress syndrome. Ann Transl Med 2017:5:295.

52. Booth CM, Rapoport B. Uptake of novel medical therapies in the general population. Curr Oncol 2011:18:105-8.

Cite this article as: Syue SH, Chang YH, Shih PJ, Lin CL, Yeh JJ, Kao CH. Polymyositis/dermatomyositis is a potential risk factor for acute respiratory failure: a pulmonary heart disease. Ann Transl Med 2020;8(5):202. doi: 10.21037/ atm.2020.01.56 